\title{
Synthesis, structure, magnetic and photoluminescent properties of dysprosium(III) Schiff Base single-molecule magnets: investigation of the relaxation of the magnetization
}

\author{
Jérôme Long, ${ }^{*[a]}$ Ivan V. Basalov,${ }^{[b]}$ Konstantin A. Lyssenko, ${ }^{[\mathrm{c}, \mathrm{d}]}$ Anton V. Cherkasov, ${ }^{[\mathrm{b}]}$ Ekaterina \\ Mamontova, ${ }^{[a]}$ Yannick Guari, ${ }^{[a]}$ Joulia Larionova ${ }^{*[a]}$ and Alexander A. Trifonov ${ }^{*[b, c]}$

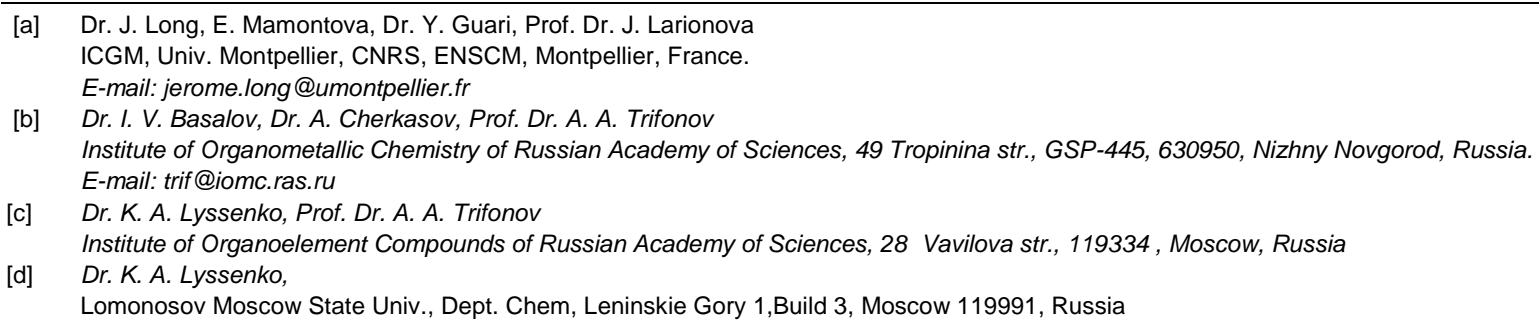

\begin{abstract}
We report here the synthesis, structure, magnetic and photoluminescence properties of three new bifunctional Schiff-base complexes $\left[\mathrm{Dy}\left(\mathrm{L}_{1}\right)_{2}(\mathrm{py})_{2}\right]\left[\mathrm{B}(\mathrm{Ph})_{4}\right] \cdot \mathrm{py}(\mathbf{1}), \quad\left[\mathrm{Dy}\left(\mathrm{L}_{1}\right)_{2} \mathrm{Cl}(\mathrm{DME})\right] \cdot 0.5 \mathrm{DME}$ (2) and $\left[\mathrm{Dy}\left(\mathrm{L}_{2}\right)_{2} \mathrm{Cl}\right] \cdot 2.5\left(\mathrm{C}_{7} \mathrm{H}_{8}\right)$ (3) $\left(\mathrm{HL}_{1}=\right.$ Phenol, 2,4-bis $(1,1-$ dimethylethyl)-6-[[(2-methoxy-5-methylphenyl)imino]methyl $] ; \mathrm{HL}_{2}=$ Phenol, 2,4-bis(1,1-dimethylethyl)-6-[[(2-methoxyphenyl)imino] methyl]). The coordination environment of the $\mathrm{Dy}^{3+}$ ion and the direction of the anisotropic axis may be controlled by the combination of the substituent groups of the Schiff bases, the nature of the counter-ions $\left(\mathrm{Cl}^{-}\right.$vs. $\left.\mathrm{BPh}_{4}{ }^{-}\right)$and the coordinative solvent molecules. A zero-field slow relaxation of the magnetization is evidenced for all complexes but strong differences in the relaxation dynamics are observed depending on the $\mathrm{Dy}^{3+}$ site geometry. In this sense, complex 1 exhibits an anisotropic barrier of $472 \mathrm{~cm}^{-1}$, which may be favourably compared to other related examples due to the shortening of the Dy-O bond in the axial direction. Besides, the three complexes exhibit a ligand-based luminescence making them as bifunctional magneto-luminescent systems.
\end{abstract}

\section{Introduction}

Lanthanide-based Single-Molecule Magnets (SMMs) are an exciting family of coordination or organometallic complexes exhibiting a slow relaxation of their magnetization, signifying that they can retain their magnetization in the absence of a magnetic field. ${ }^{[1]}$ This feature allows them to be frequently considered as pertinent candidates for future applications in data storage, spintronics or quantum computing. ${ }^{[1 a, 2]}$ These tremendous expectations however encounter difficulties associated with the relatively low maximum temperature (also known as blocking temperature) at which the magnetic information can be preserved. The physical foundation of such phenomenon is mainly correlated with the anisotropic barrier opposing the reversal of the magnetization aligned to the anisotropy axis of the complex. In mononuclear lanthanide SMMs, the control of the anisotropy may be achieved through an important axial crystal-field generated by the ligands over a specific lanthanide ion. Such acquaintanceship between $4 f$ ion and ligands could be chemically optimized in order to design complexes with targeted coordination environments, with the aim to maximize the magnetic anisotropy and reduce the rate of the Quantum Tunneling of the Magnetization (QTM) relaxation, ${ }^{[3]}$ two necessary conditions for the blocking of the magnetization. Besides QTM, intricate spin-lattice relaxations (Raman, direct) can also create underbarrier pathways. ${ }^{[4]}$ For instance, the role of molecular vibrations on the slow relaxation dynamics has been very recently recognized as a critical factor to enhance the overall properties. ${ }^{[4 a, 4 b, 5]}$ Using these concepts, considerable advances have recently been achieved in the field with several mononuclear SMMs exhibiting giant anisotropic barriers of few thousands of $\mathrm{cm}^{-1},{ }^{[3 \mathrm{~b}, 6]}$ and magnetic hysteresis that could reach liquid nitrogen's boiling point. ${ }^{[4 a, 6 c]}$

In this sense, simple electrostatic considerations could be exploited to design high anisotropic barriers SMMs. Thus, lanthanide ions with an oblate electronic $4 f$ density (flattened spheroid), such as $\mathrm{Dy}^{3+}$, could be efficiently stabilized by an axial-crystal field. It has been first demonstrated by theoretical modelling $^{[3 b, 7]}$ and then experimentally, that this could be achieved in linear complexes exhibiting two negatively charged ligands with short $\mathrm{Ln}^{3+}$-ligand distances along an axial direction that allows maximizing the crystal-field splitting, shortcutting the $\mathrm{QTM}^{[4 a, 6 c, 6 d, 8]}$ and reduce the metal-ligand vibrational modes. ${ }^{[5]}$ Additionally, the $\mathrm{Ln}^{3+}$-ligand distances in the equatorial plane should be as long as possible.

While organometallic chemistry ligands, such as cyclopentadienyl derivatives, appear particularly efficient to design highly axial systems, examples of Werner-type complexes based on ligands involving nitrogen or oxygen donors are still rather scarce owing to the difficulty to accurately control the coordination environment. ${ }^{[6 a, 6 e, 9]}$ In this regard, Schiff bases involving phenoxide moieties are among the most popular ligands to obtain lanthanide SMMs, that could eventually benefit from air-stability, ${ }^{[1 a, 1 b, 1 d, 10]}$ due to their large tunability (electronic, steric etc...) and the possibility to implement additional functionalities, such as luminescence. ${ }^{[11]}$ Consequently, an important effort has been done to rationally design high energy barrier SMMs involving such ligands. ${ }^{[6 e, 12]}$ With this in mind, we have recently reported two multifunctional dysprosium complexes based on unsymmetrical bulky tridentate $\mathrm{N}, \mathrm{N}, \mathrm{O}^{-}$ Schiff base ligands, 4-di-tert-butyl-6-((quinolin-8ylimino)methyl)phenolate and 2,4-di-tert-butyl-6-(((pyridin-2ylmethyl)imino)methyl)phenolate. They displayed a genuine SMM behaviour with relatively high energy barriers, which is also associated with a ligand-based luminescence. ${ }^{[13]}$ Encouraged by these promising results, we pursued our effort 
on the optimization of Dy ${ }^{3+}$-Schiff base SMMs and focused on the influence of the pendant group, coordinated solvent molecules and counter anion on the coordination environment of the $\mathrm{Dy}^{3+}$ and then on the magnetic properties. We report in this article the synthesis, crystal structures, magnetic and photoluminescence properties of three novel mononuclear dysprosium complexes based on two tridentate $\mathrm{N}, \mathrm{O}, \mathrm{O}^{-}$Schiff base ligands, which differ by the nature of pendant groups (the substituent by the ether oxygen (methyl vs. phenyl). Note that the steric hindrance imposed by tert-butyl groups of these ligands is favorable to generate mononuclear complexes, while the presence of different pendant groups permits to finely modify the environment of the dysprosium site and also slightly alter the emission wavelength. Moreover, the impact of the coordinated solvent molecules and the counter ions $\left(\mathrm{Cl}^{-}\right.$vs. $\left.\mathrm{BPh}_{4}{ }^{-}\right)$on the coordination environment of the $\mathrm{Dy}^{3+}$ ion and the direction of the anisotropic axes is also investigated. All compounds exhibit a slow relaxation of the magnetization associated with a ligandbased luminescence.

\section{Results and Discussion}

\section{Synthesis and crystal structures.}

The synthetic strategy relies on the combination between the oblate electronic density of $\mathrm{Dy}^{3+}$ ion with the unsymmetrical bulky tridentate ligands, $\mathrm{HL}_{1}$ (Phenol, 2,4-bis(1,1-dimethylethyl)6-[[(2-methoxy-5-methyl phenyl)imino]methyl]) or $\mathrm{HL}_{2}$ (Phenol, 2, 4-bis(1,1-dimethylethyl)-6-[[(2-methoxyphenyl)imino]methyl]), containing the donor atoms $\mathrm{N}, \mathrm{O}^{-} \mathrm{O}^{-}$. Incorporation of bulky tertbutyl groups into the ortho position to the phenolate oxygen allows limiting the coordination of "parasitic" ligands that would decrease the axiality, while variation of the substituent on the ether oxygen ( $\mathrm{Me} \mathrm{vs.} \mathrm{Ph}$ ) may affect the symmetry of the dysprosium centre. Hence, a series of cationic $\left[\mathrm{Dy}\left(\mathrm{L}_{1}\right)_{2}(\mathrm{py})_{2}\right]\left[\mathrm{B}(\mathrm{Ph})_{4}\right] \cdot \mathrm{py} \quad$ (1) and neutral $\left[\mathrm{Dy}\left(\mathrm{L}_{1}\right)_{2} \mathrm{Cl}(\mathrm{DME})\right] \cdot 0.5 \mathrm{DME} \quad(2) ; \quad\left[\mathrm{Dy}\left(\mathrm{L}_{2}\right)_{2} \mathrm{Cl}\right] \cdot 2.5\left(\mathrm{C}_{7} \mathrm{H}_{8}\right)$ complexes was synthesized using a salt metathesis approach (Scheme 1).

The reactions of anhydrous $\mathrm{DyCl}_{3}$ with 2 equivalents of in situ prepared potassium phenolates $\mathrm{KL}_{1}$ or $\mathrm{KL}_{2}$ in $\mathrm{THF}$ at room temperature afford the bis(iminophenolate)chloro dysprosium $\left[\mathrm{Dy}\left(\mathrm{L}_{1}\right)_{2} \mathrm{Cl}(\mathrm{DME})\right](2)$ or $\left[\mathrm{Dy}\left(\mathrm{L}_{2}\right)_{2} \mathrm{Cl}\right]$ (3) complexes which were isolated in 84 and $82 \%$ yields respectively.

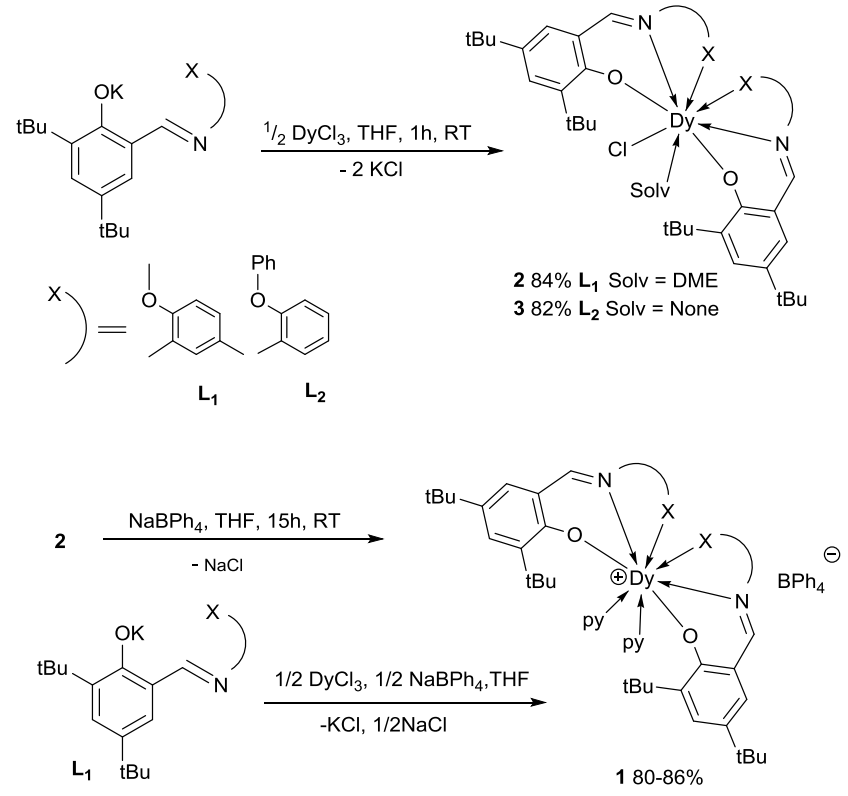

Scheme 1. Synthesis of complexes 1-3

The reaction of complex 2 with one equivalent of $\mathrm{NaBPh}_{4}$ in THF, followed by recrystallization from a THF:py:hexane mixture $(1: 1: 2)$ resulted in the formation of the cationic complex $\left[\mathrm{Dy}\left(\mathrm{L}_{1}\right)_{2}(\mathrm{py})_{2}\right]\left[\mathrm{B}(\mathrm{Ph})_{4}\right](\mathbf{1})$ in $86 \%$ yield. "One-pot" reaction of the in situ prepared potassium phenolate $\mathrm{KL}_{1}$ with $\mathrm{DyCl}_{3}$ and $\mathrm{NaBPh}_{4}$ (1:0.5:0.5 molar ratio) in THF at room temperature also allows the synthesis of $\mathbf{1}$ in $80 \%$ yield. Unfortunately, the attempts to synthesize the cationic dysprosium complex coordinated by $\mathrm{L}_{2}^{-}$ligand following the same synthetic protocol were unsuccessful.No product formation was observed when equimolar amounts of complex 3 and $\mathrm{NaBPh}_{4}$ were refluxed in THF at $70^{\circ} \mathrm{C}$ for 72 hours (Scheme 1).

$X$-ray analysis performed on single crystals indicates that 1 exists as a separated ion pair. It crystallizes in the triclinic $P \overline{1}$ space group (Table $\mathrm{S} 1$ ) with a unique $\left[\mathrm{Dy}\left(\mathrm{L}_{1}\right)_{2}(\mathrm{py})_{2}\right]^{+}$cationic moiety in the asymmetric unit. The coordination sphere of the $\mathrm{Dy}^{3+}$ is composed by four oxygens and two nitrogens belonging to two $L_{1}{ }^{-}$ligands and two nitrogen atoms from pyridine moieties giving a formal coordination number of eight for the $\mathrm{Dy}^{3+}$ ion (Figure 1). The asymmetric unit also contains a solvate pyridine molecule and a tetraphenylborate anion which ensures the electroneutrality. The Dy- $\mathrm{N}$ distances are ranging between 2.488(3) and 2.592(3) $\AA$ while the Dy-O(methoxy) ones are equal to 2.476(2) and 2.506(2) $\AA$. As expected, the shortest distances are observed for the Dy-O(phenoxide) bonds with values of 2.172(2) and 2.183(2) $\AA$ (Table 1). The bent angle $\mathrm{O}$ (phenoxide)-Dy-O(phenoxide) is equal to $124.55(9)^{\circ}$. The coordination geometry was quantitatively further analyzed with the SHAPE ${ }^{[14]}$ software which indicates a distorted biaugmented trigonal prism geometry (Table S2) whereas the shortest intermolecular $\mathrm{Dy}^{3+}-\mathrm{Dy}^{3+}$ distance found in the crystal is equal to 10.8104(4) A (Figure 2, Table 1).

Complex 2 crystallizes in the $P 2_{1} / n$ space group. The asymmetric part of the unit cell in $\mathbf{2}$ contains one dysprosium complex and a DME solvate molecule located on an inversion centre giving the resulting formula $\left[\mathrm{Dy}(\mathrm{L} 1)_{2} \mathrm{Cl}(\mathrm{DME})\right] \cdot 0.5 \mathrm{DME}$. In contrast to $\mathbf{1}$, the coordination sphere of the $\mathrm{Dy}^{3+}$ in $\mathbf{2}$ along with two $\mathrm{L}_{2}^{-}$ligands, contains one chloride and a monodentate DME 
ligands resulting also in a formal coordination number of eight (Figure 1). Similarly to $\mathbf{1}$, the shortest distances accounts for the Dy-O(phenoxide) with values of 2.185(6) and 2.223(5) $\AA$ while the $\mathrm{Dy}-\mathrm{Cl}$ one is expectedly significantly larger and equal to 2.621(2) ^ (Table 1). The O(phenoxide)-Dy-O(phenoxide) angle is noticeably larger than in 1 with a value of $144.6(2)^{\circ}$. The shortest interomolecular $\mathrm{Dy}^{3+}-\mathrm{Dy}^{3+}$ distance in 2 is equal to 9.2237(5) Å (Figure 2).
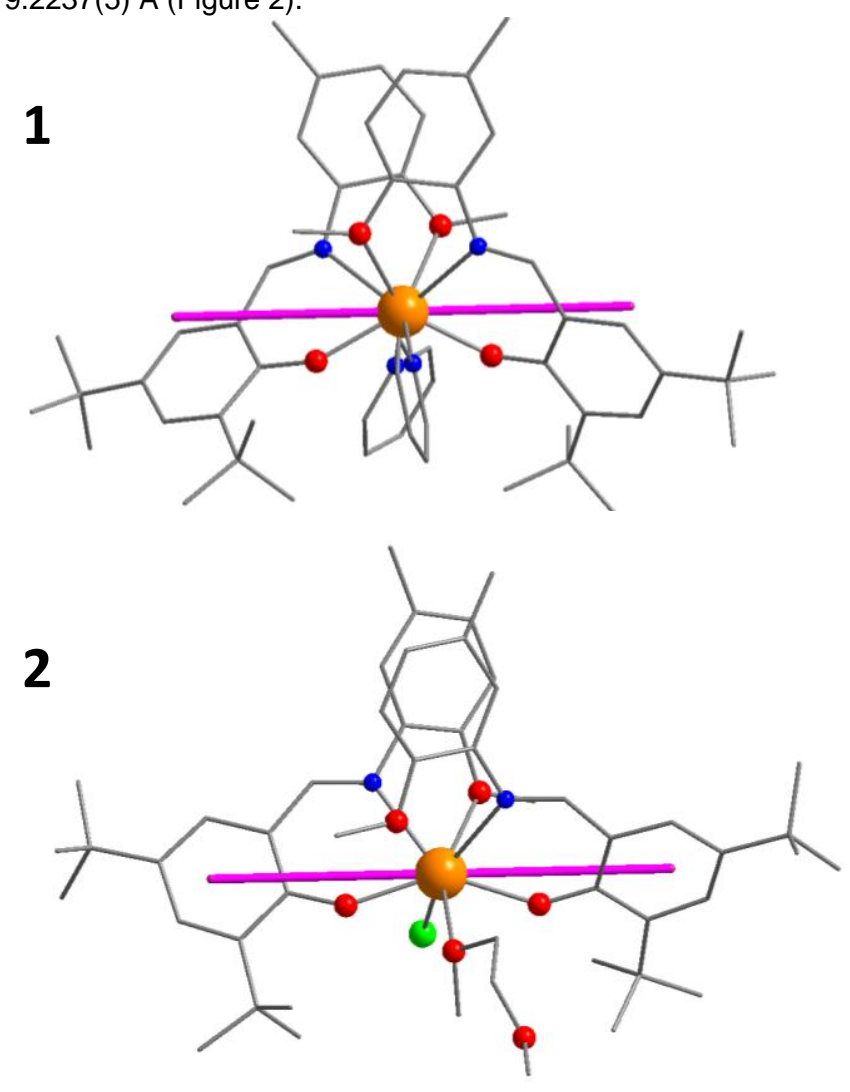

3

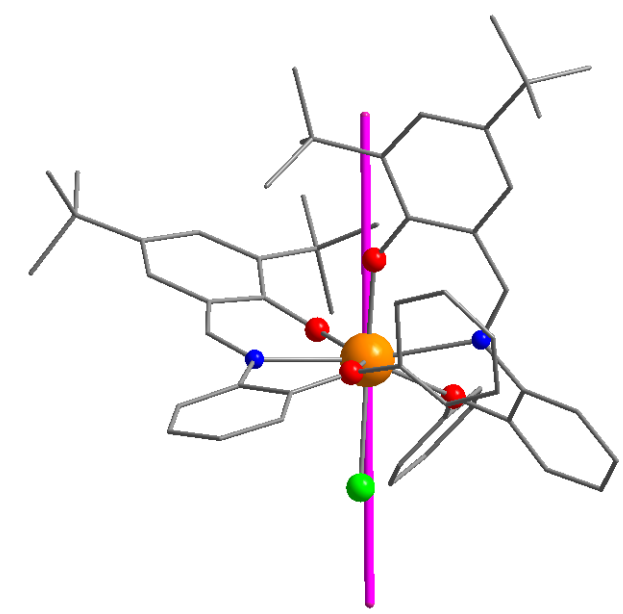

Figure 1. Molecular structure for complexes 1-3. Colour code: orange, Dy; blue, $\mathrm{N}$; grey, $\mathrm{C}$; green, $\mathrm{Cl}$; Hydrogen atoms, $\left[\mathrm{B}(\mathrm{Ph})_{4}\right]^{-}$and solvate molecules have been omitted for clarity. The purple lines represent the anisotropic axes for the ground doublet obtained with MAGELLAN. ${ }^{[15]}$

Finally, compound 3 crystallizes as a toluene solvate in the triclinic $P \overline{1}$ space group with one dysprosium complex in the asymmetric unit.
1)

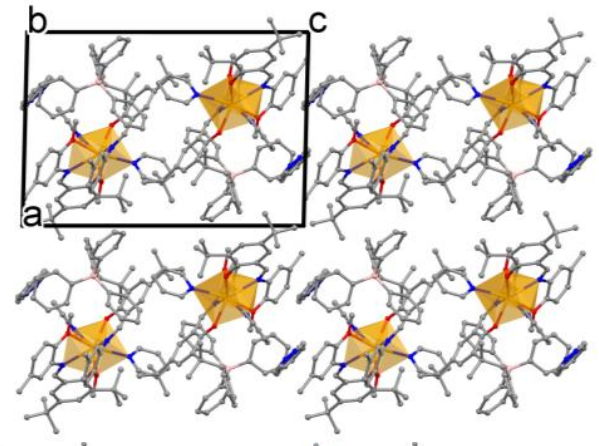

2)

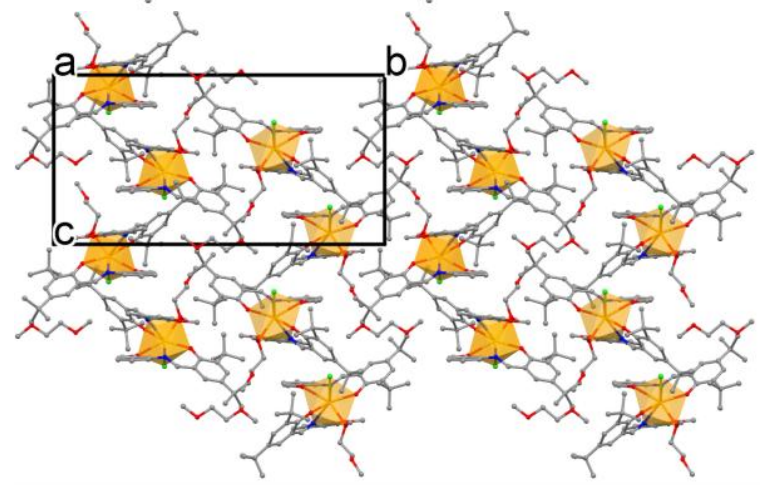

3)

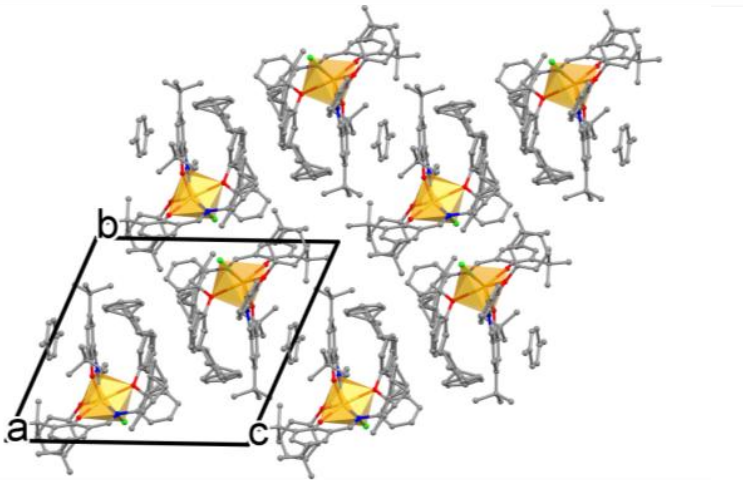

Figure 2. Perspective views of the crystal packing for 1-3 showing the coordination polyhedra.

Table 1. Selected bond lengths and angles in complexes 1-5.

\begin{tabular}{|c|c|c|c|c|c|}
\hline Compound & $\begin{array}{c}\text { Dy-O- } \\
\text { phenoxide } \\
\text { distances } \\
(\AA \AA)\end{array}$ & $\begin{array}{l}\mathrm{O}^{-} \text {-Dy-O- } \\
\text { angle }\left({ }^{\circ}\right)\end{array}$ & $\begin{array}{c}\text { Dy-Cl } \\
\text { distances } \\
(\AA ̊)\end{array}$ & $\begin{array}{c}\text { Dy-Dy } \\
\text { intermolecular } \\
\text { distance } \\
(\AA)\end{array}$ & Ref. \\
\hline 1 & $\begin{array}{l}2.172(2) \\
2.183(2)\end{array}$ & $124.55(9)$ & - & $10.8104(4)$ & $\begin{array}{l}\text { This } \\
\text { work }\end{array}$ \\
\hline 2 & $\begin{array}{l}2.185(6) \\
2.223(5)\end{array}$ & $144.6(2)$ & $2.621(2)$ & $9.2237(5)$ & $\begin{array}{l}\text { This } \\
\text { work }\end{array}$ \\
\hline 3 & $\begin{array}{l}2.139(2) \\
2.143(2)\end{array}$ & $89.76(9)$ & $2.6369(9)$ & $7.8396(6)$ & $\begin{array}{l}\text { This } \\
\text { work }\end{array}$ \\
\hline 4 & $2.190(6)$ & $128.5(2)$ & - & 13.9590 & [13] \\
\hline 5 & $\begin{array}{l}2.193(2) \\
2.196(2)\end{array}$ & $121.20(4)$ & - & $10.6582(8)$ & [13] \\
\hline
\end{tabular}

The structure is reminiscent to that of $\mathbf{2}$ with the exception that the absence of a coordinated solvent molecule gives an heptacoordinated geometry for the $\mathrm{Dy}^{3+}$ site (Figure 1), close to 
a distorted capped octahedron (Table S3). The two DyO(phenoxide) distances are equal to 2.139(2) and 2.143(2) $\AA$. In contrast to 2, the bent angle $\mathrm{O}$ (phenoxide)-Dy-O(phenoxide) is much narrower with a value of $89.76(9)^{\circ}$. The shortest $\mathrm{Dy}^{3+}-\mathrm{Dy}^{3+}$ distance is found equal to 7.8396(6) $\AA$ (Figure 2). In comparison with the related complexes based on tridentate $\mathrm{N}, \mathrm{N}, \mathrm{O}^{-}$ligands we recently reported ${ }^{[13]}$ (denoted as 4 and 5 , Table 1), the distances and the angles are summarized in Table 1.

\section{Magnetic Properties.}

The magnetic properties of the three complexes were investigated by SQUID magnetometry.

Firstly, the direct current (dc) magnetic properties were analyzed. At room temperature, the $\chi^{T}$ values of $13.74,14.55$ and 13.32 $\mathrm{cm}^{3} . \mathrm{K} . \mathrm{mol}^{-1}$ for $\mathbf{1 , 2}$ and $\mathbf{3}$ respectively are in a relatively good agreement with the value of $14.17 \mathrm{~cm}^{3} . \mathrm{K} \mathrm{mol}^{-1}$ expected for a single $\mathrm{Dy}^{3+}$ ion using the free-ion approximation (Figure 3 ). Compounds 2 and $\mathbf{3}$ show a continuous decrease of $\chi T$ upon cooling originating from the thermal depopulation of the $m_{J}$ levels. In contrast, the $\chi^{T}$ product for compound $\mathbf{1}$ remains nearly constant as the temperature is reduced and then exhibits a dramatic decrease at low temperature suggesting a larger crystal-field splitting with respect to 2 and 3 . At $1.8 \mathrm{~K}$, the magnetization values under a $70 \mathrm{kOe}$ field are 5.82, 4.53 and $5.25 N \beta$ for 1, 2 and $\mathbf{3}$ respectively (Figure 3). For complex 1, a clear inflection point could be observed at low field. Such trend

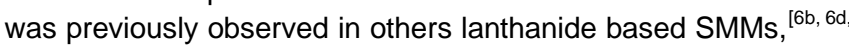
${ }^{13,16]}$ and may reflect either the extreme field dependence of the relaxation rates ${ }^{[9 b]}$, the flip of the $D y^{3+}$ spins under an applied magnetic field. ${ }^{[16]}$

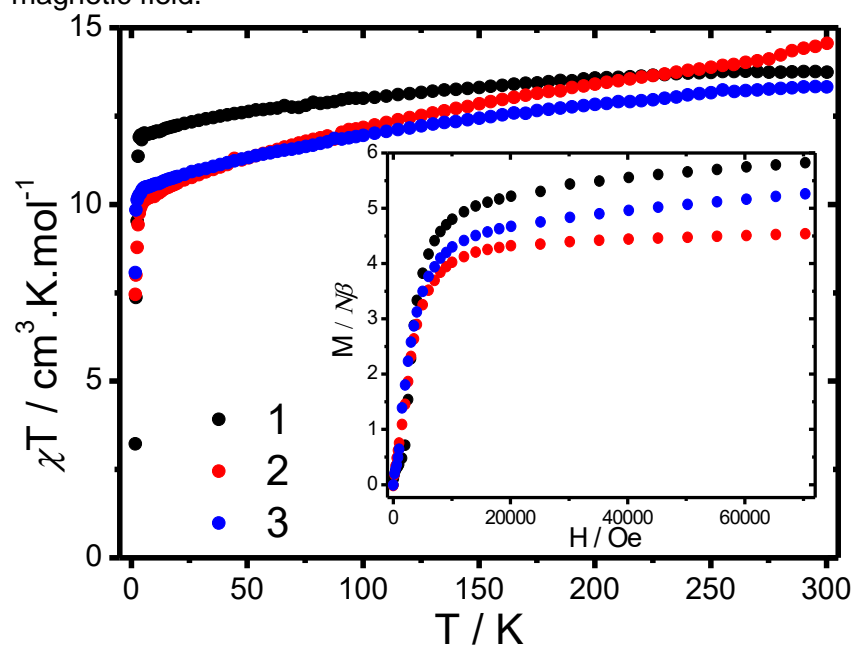

Figure 3. Temperature dependence of $\chi T$ under an applied magnetic field of 1000 Oe for 1-3. Inset: Field dependence of the magnetization at $1.8 \mathrm{~K}$.

Alternate currents (ac) measurements were then performed in order to characterize the occurrence of a SMM behaviour. Under a zero dc-field, the three complexes exhibit an important signal of the out-of-phase $\left(\chi^{\prime \prime}\right)$ of the ac susceptibility indicating the presence of a slow relaxation of the magnetization (Figure 4, Figure S1). Nevertheless, important differences are readily observed. Hence, the frequency dependences of $\chi^{\prime \prime}$ exhibits a single temperature dependent peak for $\mathbf{1}$ and $\mathbf{3}$ whereas a peak associated with a plateau at high frequencies is observed for 2. Moreover, the temperature range of the slow relaxation greatly differs (Figure 1, Figure S1). Thus, out-of phase signals are observed up to 45 and $15 \mathrm{~K}$ for 2 and 3 respectively, while it reaches up $60 \mathrm{~K}$ for 1 (Figure S2).

The Cole-Cole plots for 1 (Figure S3) confirm the occurrence of a single relaxation for $\mathbf{1}$ and could be fitted with a generalized Debye model, leading to low values of the $\alpha$ parameter $(<0.1)$ in the high temperature region while it significantly increases (up to 0.5) at low temperature (Table S4). Surprisingly, the Cole-Cole plots for $\mathbf{3}$ at low temperature could be only badly fitted with a single function suggesting a very wide distribution of relaxation times (Figure S3, Table S6). Although such phenomenon could have various origins, one can note a slight crystallographic disorder observed on one $\mathrm{L}_{2}^{-}$ligand. As regards 2, the presence of a low temperature second relaxation process could be ultimately confirmed by the Cole-Cole plots (Figure S3) which could be fitted with a sum of two modified Debye functions with $\alpha$ parameters lower than 0.36) (Table S5).

In order to obtain further insights into the relaxation dynamics, the temperature dependence of the relaxation time, $\tau$, was monitored for the three complexes. The classical deviation from the linearity observed in the $\ln \tau$ vs. $T^{-1}$ plot (Figure 5) indicates an overlap between different relaxation processes.

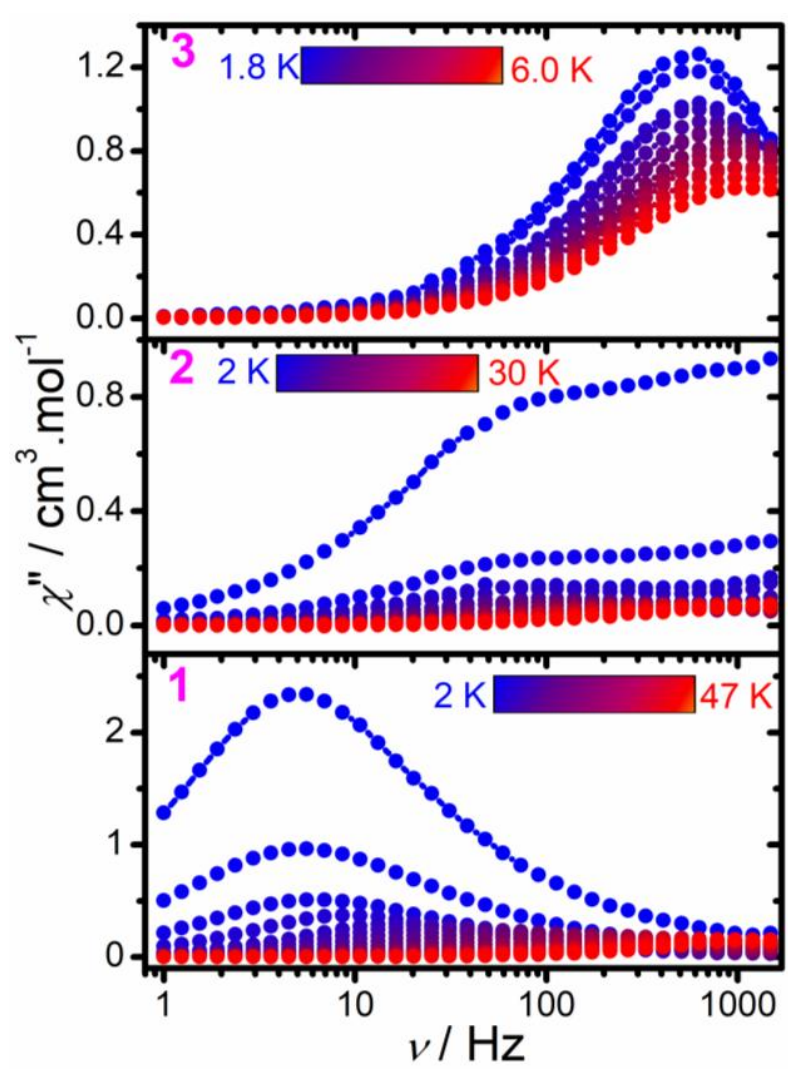

Figure 4. Frequency dependence of $\chi^{\prime \prime}$ for compounds 1-3 under a zero dcfield.

Consequently, the fitting of the whole temperature range could be achieved using the following model: $\tau^{-1}=\tau_{0}{ }^{-1} \exp (-\Delta / k T)+$ $C T^{n}+\tau^{-1}$ QTM (Eq. 1). ${ }^{[17]}$ The first term accounts for a thermally activated process, while the second and third ones stand for two-phonon Raman and QTM, respectively. In order to avoid over-parameterization, the $n$ coefficient was successively fixed to various values until getting the best correlation coefficient. The obtained fitting parameters could be found in Table 2 and 
indicate that, for complex $\mathbf{1}$, the relaxation involves the three considered processes. Interestingly, the obtained value of $\Delta=$ $472 \pm 25 \mathrm{~cm}^{-1}$ is found much larger than in our two related previous examples based on $\mathrm{N}, \mathrm{N}, \mathrm{O}^{-}$Schiff base ligands (Table 2). ${ }^{[13]}$ In contrast, meaningless parameters are obtained for complex 2 using Eq. 1. Nevertheless, performing the fit taking only into account Raman and QTM processes ( $n$ being let free) yields to better results. The $n$ value of 4.3 is close to the value of 5 , which is sometimes observed for Kramers ion. ${ }^{[18]}$ Due to the wide distribution of the relaxation times, the situation for complex 3 is more complicated and it was not possible to perform any pertinent fitting. Moreover, the presence of strong dipolar interactions caused by short intermolecular $\mathrm{Dy}^{3+}-\mathrm{Dy}^{3+}$ distances (i.e. $7.840 \AA$ ) may also complicate the overall picture. Nevertheless, it could be noticed that the relaxation time is found several order of magnitude lower with respect to complexes 1 and 2. As a consequence, dramatic differences in the zero-field relaxation dynamics could be observed.

Table 2. Fit parameters of the temperature dependence of the relaxation time for $1,2,4$ and 5 .

\begin{tabular}{|c|c|c|c|c|c|}
\hline Compound & $\Delta\left(\mathrm{cm}^{-1}\right)$ & $\tau_{0}(s)$ & $n$ & $C\left(\mathrm{~s}^{-1} \cdot \mathrm{K}^{-n}\right)$ & $\begin{array}{l}\tau_{\text {QTM }} \\
(m s)\end{array}$ \\
\hline $1(0 \mathrm{Oe})$ & $472 \pm 25$ & $\begin{array}{c}(2 \pm 1) \times \\
10^{-10}\end{array}$ & $5.35^{\star}$ & $\begin{array}{c}(6.2 \pm 0.1) \times \\
10^{-6}\end{array}$ & $\begin{array}{c}20 \pm \\
3\end{array}$ \\
\hline $2(0 \mathrm{Oe})$ & - & - & $4.3 \pm 0.2$ & $\begin{array}{c}0.0012 \pm \\
0.0008\end{array}$ & $\begin{array}{c}2.6 \pm \\
0.2\end{array}$ \\
\hline $4(0 \mathrm{Oe})$ & $272 \pm 5$ & $\begin{array}{c}(4.1 \pm \\
0.6) \times \\
10^{-8}\end{array}$ & $3^{*}$ & $\begin{array}{c}0.0157 \pm \\
0.0008\end{array}$ & $\begin{array}{c}14 \pm \\
2\end{array}$ \\
\hline $5(0 \mathrm{Oe})$ & $294 \pm 4$ & $\begin{array}{c}(6.6 \pm \\
0.8) \times \\
10^{-9}\end{array}$ & $3^{*}$ & $\begin{array}{c}0.0231 \pm \\
0.0009\end{array}$ & $\begin{array}{c}24 \pm \\
7\end{array}$ \\
\hline
\end{tabular}

*fixed parameter

In order to shortcut the contribution of the QTM, the field dependence of the ac susceptibilities and in turn the relaxation time were monitored (Figures S4-S5). Upon applying a dc field larger than $500 \mathrm{Oe}$, the three complexes exhibit a relaxation time which remains constant in the investigated field range. A dc field value of 1000 Oe was therefore chosen as the optimum field for all samples.

To our knowledge, the qualitative description of the dynamics of Raman process in SMMs is mainly based on physic concepts derived from EPR spectroscopy on simple inorganic salts.

On one hand, some studies have reported that the fieldindependent character of the Raman process ${ }^{[19]}$ while others have predicted a complex field dependence as $\tau^{-1}=C \frac{1+C_{1} H^{2}}{1+C_{2} H^{2}} T^{n}$ (for Kramers ions). ${ }^{[20]}$

Some recent studies have highlighted that while the first order Raman process is field independent, the second order Raman process implies a field-dependence. ${ }^{[21]}$ Considering the field dependent character of the Raman process will require fitting the field dependence of the relaxation time with eight different parameters which will undoubtedly lead to over-parameterization and meaningless results. Besides, recent studies have also anticipated or reported that the Raman relaxation is independent on the magnetic field strength in SMMs based on Kramers

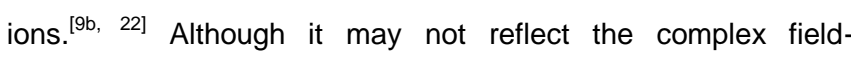
dependence of the Raman process, we have integrated the Raman contribution in the $K$ constant with the Orbach process Hence, the field dependence of the relaxation time (Figure S5) was modeled using: $\tau^{-1}=\frac{B_{1}}{1+B_{2} H^{2}}+D H^{4} T+K$ (Eq. 2),

The increased values of $B_{1}$ and $B_{2}$ going from complexes 1 to 3 (Table S7) directly reflects the increase of the QTM rate. As a consequence, the frequency dependences of the ac susceptibilities under a dc field of 1000 Oe (Figure S6) validate the decrease of the QTM. It could be noticed that for 3, applying dc fields greatly shifts the slow relaxation temperature range for which maximum of $\chi^{\prime \prime}$ could be observed up to $14.5 \mathrm{~K}$. The ColeCole plots (Figure S7) further corroborate the presence of a second relaxation for complex 2 at low temperature. Remarkably, the fitting of the Cole-Cole plots with either a generalized Debye model (complexes $\mathbf{1}$ and $\mathbf{3}$ ) or the sum of two modified Debye functions (complex 2) (Tables S8-S10) yield to weak values for the $\alpha$ parameter $(\alpha<0.03)$ for the three complexes. This indicates that applying a dc-field causes a dramatic influence over the distribution of relaxation times with respect to the zerofield data. Such results imply that the large distribution of the relaxation times is somehow related to the QTM process and in some extent to the crystallographic disorder or an anisotropic displacement of the coordinated atoms in the coordination sphere of the $\mathrm{Dy}^{3+}$, as it was recently suggested. ${ }^{[23]}$ Fitting of the temperature dependence for 2 and 3 could be carried out considering only a Raman process as $\tau^{-1}=C T^{n}$ (Table S11, Figure S8). On the other hand, the fitting for 1 was performed with the following model with $\tau^{-1}=\tau_{0}^{-1} \exp (-\Delta / k T)+C T^{n}$ (Eq. 3) (Figure S8, Table S11). As expected for a thermally activated process, the anisotropic barrier is found comparable, taken into account the uncertainties of the fitting, with respect to that obtained under a zero-dc field.

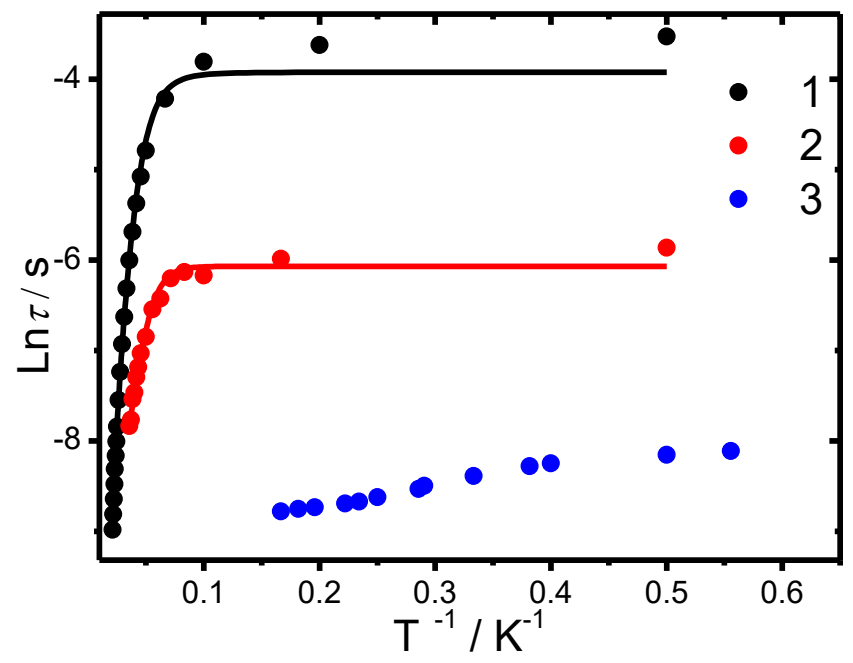

Figure 5. Temperature dependence of the relaxation time, $\tau$, for complexes 13 under a zero dc-field. The solid lines represent the fit.

Obviously, complex 1 exhibits the most interesting magnetic properties. In this regards, it has been recently highlighted that large $\alpha$ parameter values may result in large uncertainties for the corresponding relaxation times. ${ }^{[23]}$ In order to qualitatively compare the anisotropic barriers, the uncertainties on the relaxation time $\tau$ for compounds $\mathbf{1 , 4}$ and $\mathbf{5}$ have been calculated 
from the $\alpha$ parameters of the generalized Debye model using the CC-Fit2 software based on the log-normal distribution. ${ }^{[23]}$ Figure S9 clearly shows the strong decrease of the $\tau$ uncertainties for compound $\mathbf{1}$ for the data collected under a dc-field. Starting from this, we have reinvestigated the fitting of the relaxation times for 4 and 5 (Table 2, Figure S10). The slightly different obtained parameters with respect our previous study ${ }^{[13]}$ could be rationalized from the different methods of the extraction of the relaxation times (i.e. using CC-Fit2). Similarly than for compound $\mathbf{1}$, the uncertainties related to $\tau$ for 5 are found greatly reduced for the in-field data with respect to those obtained under a zerodc field, whereas this decrease is less obvious for 4 (Figure S10). Since the thermally activated process is field-independent, these results indicate that the in-field data should give a more accurate estimation of the anisotropic barriers, in accordance with the trend observed in zero-dc field. Consequently, while for $\mathbf{4}$ and $\mathbf{5}$, the $\Delta$ values are found comparable (within the uncertainties), complex 1 shows clearly a greater barrier.

Finally, the ability of lanthanide SMMs to maintain their magnetization constitutes definitely an important feature for future applications. Among the three new investigated complexes, $\mathbf{1}$ and $\mathbf{2}$ clearly exhibits the more probabilities to present a clear magnetic bistability. For comparison, the relaxation time for complex 1 at $2 \mathrm{~K}$ is found almost 70 times greater with respect to $\mathbf{3}$. The Zero-Field Cooled (ZFC) and Field-Cooled (FC) curves reveal distinct behaviours. While a clear maximum in the ZFC curve (one of the possible definition for the blocking temperature $T_{\mathrm{B}}{ }^{[24]}$ ) is observed for 1 at $2.6 \mathrm{~K}$, only a slight non-superposition between the ZFC and FC curves is detected for 2 (Figure S11). This suggests that 1 exhibits a higher blocking temperature. The divergence between the ZFC/FC curves occurs at 6.0 and $5.5 \mathrm{~K}$ for 1 and 2 respectively. This point could be described as an irreversible temperature $\left(T_{\text {irr. }}\right)$ and reflects an out-of-equilibrium state frequently observed in lanthanide SMMs. ${ }^{[4,6 c, 6 d, 9 a, 25]}$ Moreover, the magnetic irreversibility is further confirmed by the opening in the hysteresis loops for $\mathbf{1}$ and $\mathbf{2}$ at $1.8 \mathrm{~K}$ (Figure 6). For complex $\mathbf{1}$ which clearly shows the best performances, the hysteresis loop is almost closed at $5 \mathrm{~K}$ in our experimental conditions (Figure S12).

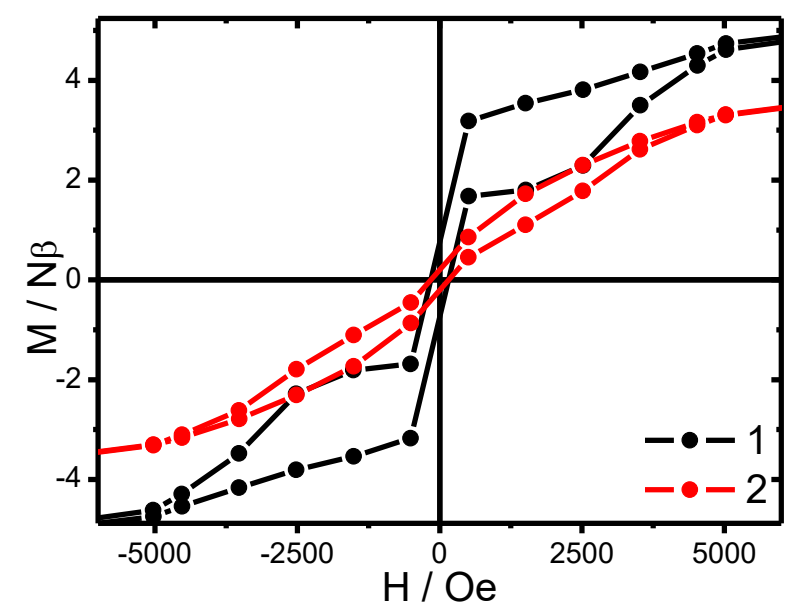

Figure 6. Hysteresis loops at $1.8 \mathrm{~K}$ for $\mathbf{1}$ and $\mathbf{2}$ with an average sweep rate of 15 Oe.s ${ }^{-1}$.

\section{Magneto-structural correlations.}

Rationalization of the observed magnetic properties could be obtained with magneto-structural correlations. It is well known that dysprosium-based SMMs show a strong dependence of their slow relaxation features depending on the coordination environment of the lanthanide site ${ }^{[3 \mathrm{~b}, 26]}$ which could be modulated by nature of the pendant group of the ligands, the nature of the counter anion and the coordinated solvent molecules. ${ }^{[27]}$

Firstly, compounds 1 and 2 are based on the same Schiff base $L_{1}{ }^{-}$ligand bearing a methyl on the ether group. While 1 is a cationic complex $\left[\mathrm{Dy}\left(\mathrm{L}_{1}\right)_{2}(\mathrm{py})_{2}\right]^{+}, 2$ is a neutral complex $\left[\mathrm{Dy}\left(\mathrm{L}_{1}\right)_{2} \mathrm{Cl}(\mathrm{DME})\right]$ incorporating a coordinated chloride anion in the coordination sphere of the $\mathrm{Dy}^{3+}$ ion. The O(phenoxide)-Dy$\mathrm{O}$ (phenoxide) (abbreviated as $\mathrm{O}^{-}-\mathrm{Dy}-\mathrm{O}^{-}$) arrangement is much greater for 2 with respect to 1 (144.8 vs. $\left.124.6^{\circ}\right)$ but the Dy-O are shorter in $\mathbf{1}$. The orientation of the anisotropic axes estimated from a simple electrostatic model with the Magellan ${ }^{[15]}$ package shows that, for both complexes, these axes are almost collinear to the $\mathrm{O}^{-}-\mathrm{Dy}-\mathrm{O}^{-}$sequence with deviation of $27.34 / 28.09^{\circ}$ and $16.84 / 18.36^{\circ}$ for 1 and 2 , respectively (Figure 1). The fact that the anisotropic axis is only moderately tilted in $\mathbf{2}$, despite the presence of the coordinated $\mathrm{Cl}^{-}$, may be explained by the relatively long Dy-Cl distance of $2.623 \AA$ that limits its electrostatic influence. However, the magnetic analysis suggests that the substitution of pyridine moieties by a chloride and DME solvent alters the relaxation dynamics. It would be tempting to correlate this with a modification in the metal-ligand vibrational modes that affects the Raman process but would require detailed far-infrared and/or Raman studies. ${ }^{[28]}$

Secondly, compounds 2 and $\mathbf{3}$ present slightly different coordination environments which vary by the nature of the ether substituents (methoxy vs. phenoxy) of the Schiff base, as well as from the solvent molecule present in the coordination sphere of $\mathrm{Dy}^{3+}$. Due to the steric hindrance brought by the phenyl groups, the coordination geometry around the $\mathrm{Dy}^{3+}$ ion for $\mathbf{3}$ is found reduced with an heptacoordinated geometry $\left[\mathrm{Dy}\left(\mathrm{L}_{2}\right)_{2} \mathrm{Cl}\right]$. Hence, a dramatically weaker $\mathrm{O}^{-}-\mathrm{Dy}-\mathrm{O}^{-}$angle of almost $90^{\circ}$ is observed for 3. In that case, the anisotropic axis obtained from Magellan is oriented along the $\mathrm{O}^{-}$-Dy- $\mathrm{Cl}$ sequence (Figure 1) since this latter provides an angle of $158^{\circ}$, closer to the linearity. Despite the fact that 3 exhibits the shortest Dy- $\mathrm{O}^{-}$distances, the presence of a negatively charged phenoxide coordinated perpendicular to the anisotropic axis induces a strong transverse component. Due to the large distribution of relaxation time at zero-field, it was not possible to study in details the zero-field relaxation dynamics for 3. However and in the same way than for 2 , the ac data obtained under a dc field suggest that it is dominated by a Raman process. Both the strong QTM and Raman relaxation explain the fact that, among the three investigated compounds, $\mathbf{3}$ shows the weakest magnetic features.

The results obtained for complex $\mathbf{1}$ may be directly compared with the ones found for our two previously reported cationic complexes $\mathbf{4}$ and $\mathbf{5}$, based on slightly different N,N,O Schiff base ligands, but for which the orientation of the anisotropic axes were found similar. ${ }^{[13]}$ Indeed, the anisotropy barrier found for $\mathbf{1}$ is much greater (see Table 2). Although the $\mathrm{O}^{-}$-Dy-O $\mathrm{O}^{-}$angles are found nearly similar $\left(124.6^{\circ}\right.$ for $1 \mathrm{vs} 128.5^{\circ}$ for 4), a slightly shorter $\mathrm{Dy}^{-\mathrm{O}^{-}}$distance of $2.173(3) \AA$ is observed in 1, with respect to the Dy- $\mathrm{O}^{-}$distances ranging from 2.190 (6) to 2.196(2) $\AA$ observed in $\mathbf{4}$ and 5. Visibly, this $0.02 \AA$ 
difference in bond length involving the negatively charged donor atom may affect the crystal-field splitting and highly enhance the magnetic axiality, which explains the important improvement of the energy barrier. ${ }^{[3 b]}$ Such increase in the anisotropic barriers with comparable bond shortening magnitude has been previously evidenced in the dysprosium metallocenium family SMMs with enlargement up to $20 \% .^{[5 b, 29]}$ In the case of Schiffbase phenoxide complexes, the highest measured anisotropic barrier of $665 \mathrm{~cm}^{-1}$ to our knowledge shows a shortest Dy- $\mathrm{O}^{-}$of $2.126 \AA$ associated with a $\mathrm{O}^{-}$-Dy- $\mathrm{O}^{-}$angle of $144.7^{\circ} .^{[6 \mathrm{e}]}$ This clearly shows that the bond distance and to a lesser extent the $\mathrm{O}^{-}$-Dy-O $\mathrm{O}^{-}$angle have to be carefully controlled in order to achieve large energy barrier systems.

Photoluminescence. Schiff base ligands are also promising to implement photoluminescence properties through the ligandbased luminescence or through the enhanced $\mathrm{Ln}^{3+}$ emission thanks to the antenna effect and therefore to design bifunctional SMM systems. ${ }^{[11]}$ The photoluminescent properties were therefore investigated in solid-state for all complexes. The room temperature emission spectra, excited at $370 \mathrm{~nm}$, show a broad emission band centered at 563, 583 and $544 \mathrm{~nm}$ for complexes 1, 2 and 3, respectively (Figure 7) while the excitation spectra (Figure S13) exhibit a large band around $370-380 \mathrm{~nm}$. The broadness of the emission band clearly indicates a ligand-based luminescence. This is confirmed by the photoluminescence spectra of $\mathrm{HL}_{1}$ and $\mathrm{HL}_{2}$ which show a comparable profile with a maximum emission wavelength at about $570 \mathrm{~nm}$ (Figure S14). Although based on the same $\mathrm{L}_{1}{ }^{-}$ligand, the differences in the emission wavelengths between $\mathbf{1}$ and $\mathbf{2}$ may be ascribed to modifications in the ligand-ligand interactions within the crystal structures. Noticeably, the three compounds do not show dysprosium-based luminescence indicating that $\mathrm{L}_{1}{ }^{-}$and $\mathrm{L}_{2}{ }^{-}$ ligands are not active as antenna for lanthanide excitation. Such behavior was also observed for complexes 4 and $5^{[13]}$

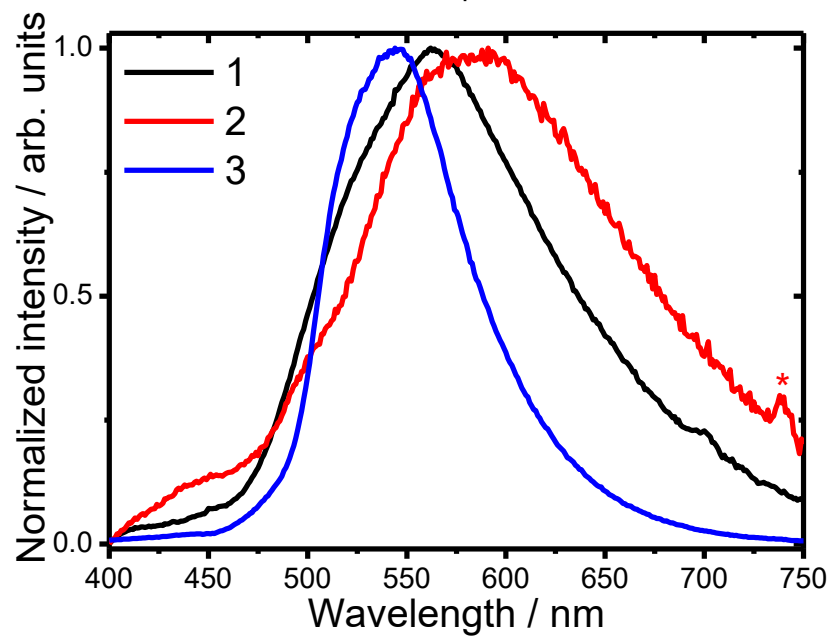

Figure 7. Room temperature normalized emission spectra for complexes 1-3 obtained under an excitation at $\lambda_{\text {exc }}=370 \mathrm{~nm}$. The asterisk sign indicates excitation second order.

\section{Conclusion}

Three new mononuclear dysprosium Schiff-base complexes with a tunable coordination environment were synthesized by the combination of bulky unsymmetrical tridentate Schiff-base ligands with coordinative solvents and counter anions. The control of the coordination environment may be achieved by playing on : i) the nature of the substituents of both, the phenol ring and ether groups, ii) the use of two different counter-anions, namely coordinative and small $\mathrm{Cl}^{-}$ion or non-coordinative and bulky $\mathrm{BPh}_{4}^{-}$, and iii) the coordination (or not) of solvent molecules through adjustment of the experimental conditions. The three reported complexes exhibit a zero-field slow relaxation of the magnetization with distinct relaxation dynamics. Hence, using the $\mathrm{L}_{1}{ }^{-}$ligand, two dysprosium complexes differing mainly by the presence of a coordinated chloride in the coordination sphere of the $\mathrm{Dy}^{3+}$ ion have been obtained. The cationic complex 1 shows a genuine SMM behavior with out-of-phase signals observed up to $60 \mathrm{~K}$ and a fair energy barrier of $472 \mathrm{~cm}^{-1}$. In comparison with the previously reported similar complexes and based on related Schiff base ligands presenting closed coordination environment and similar orientation of the anisotropic axis, the shortening of the Dy-O(phenoxide) induces an enhancement of the height of the energy barrier. On the other hand, the introduction of a coordinated chloride in the coordination sphere in complexes $\mathbf{2}$ and $\mathbf{3}$ strongly affects the relaxation dynamics that appears to follow a Raman temperature dependence. Additionally, the greater steric hindrance of the phenyl group in $\mathrm{L}_{2}^{-}$decreases severely the O-Dy-O angle. Moreover, all the complexes exhibit a ligand-centered luminescence. The great flexibility in the nature of the substituents of both, phenol and ether groups of the Schiff base ligands associated with the careful use of the counter anions and the coordinated solvents is highly promising to design genuine luminescent SMMs with high anisotropic barriers.

\section{Experimental Section}

\begin{abstract}
Materials and Methods.
All experiments were performed in evacuated tubes or in inert atmosphere, using standard Schlenk-flask or glove-box techniques, with rigorous exclusion of traces of moisture and air. After drying over $\mathrm{KOH}$, THF was purified by distillation from sodium/benzophenone ketyl, hexane and toluene by distillation from sodium/triglyme benzophenone ketyl prior to use. Pyridine was dried over $\mathrm{KH}$ and was purified by distillation prior to use. $\mathrm{L}_{1} \mathrm{H}$ and $\mathrm{L}_{2} \mathrm{H}$ were synthesized according to literature procedures. ${ }^{[30]}$ IR spectra were recorded as Nujol mulls on a Bruker-Vertex 70 spectrophotometer. The N, C, H elemental analyses were carried out in the microanalytical laboratory of the IOMC by means of a Carlo Erba Model 1106 elemental analyser with an accepted tolerance of 0.4 unit on carbon $(\mathrm{C})$, hydrogen $(\mathrm{H})$, and nitrogen $(\mathrm{N})$. Lanthanide metal analysis was carried out by complexonometric titration. ${ }^{[31]}$
\end{abstract}

\section{Synthesis of the complexes}

Synthesis of $\left[\mathrm{Dy}\left(\mathrm{L}_{1}\right)_{2}(\mathrm{py})_{2}\right]\left[\mathrm{B}(\mathrm{Ph})_{4}\right] \cdot \mathrm{py}(\mathbf{1})$.

Method A: A mixture of $\mathrm{HL}_{1}(0.200 \mathrm{~g}, 0.566 \mathrm{mmol})$ and $\mathrm{KH}(0.029 \mathrm{~g}$ $0.725 \mathrm{mmol})$ in THF (30 mL) was stirred at room temperature for $1 \mathrm{~h}$ and the resulting suspension was filtered. The clear yellow solution was added to a suspension of $\mathrm{DyCl}_{3}(0.076 \mathrm{~g}, 0.283 \mathrm{mmol})$ in THF $(10 \mathrm{~mL})$ and the reaction mixture was stirred another $1 \mathrm{~h}$. After that $\mathrm{NaBPh}_{4}$ $(0.097 \mathrm{~g}, 0.283 \mathrm{mmol})$ was added to the solution and the reaction mixture was stirred overnight at room temperature. The solution was filtered, the 
volatiles were removed in vacuum and the solid residue was redissolved in a THF:Py:Hexane mixture (1:1:2). Slow concentration of the solution at room temperature resulted in the formation of 1 as yellow crystals. The mother liquid was decanted and the crystals were washed with cold hexane and dried in vacuum for $30 \mathrm{~min}$. Complex 1 was isolated in $80 \%$ yield $(0.322 \mathrm{~g})$. Elemental analysis calcd. (\%) for $\mathrm{C}_{85} \mathrm{H}_{95} \mathrm{BDyN}_{5} \mathrm{O}_{4}$ : $\mathrm{C}$, 71.69; H, 6.73; N, 4.91; Dy, 11.41. found (\%): C, 71.99; H, 6.48; N, 5.18; Dy, 11.25. IR (Nujol, KBr) v/cm ${ }^{-1}: 490$ (s), 540 (w), 570 (w), 630 (s), 780 (s), 800 (s), 840 (s), 880 (s), 985 (m), 1030 (s), 1070 (s), 1160 (s), 1200 (m), 1230 (s), 1320 (s), 1420 (s), 1580 (s), 1600 (s) 1625 (s).

Method B: $\mathrm{NaBPh}_{4}(0.061 \mathrm{~g}, 0.180 \mathrm{mmol})$ was added to a solution of 1 $(0.178 \mathrm{~g}, 0.171 \mathrm{mmol})$ in THF $(10 \mathrm{~mL})$ and the reaction mixture was stirred at room temperature for $15 \mathrm{~h}$. The solution was filtered, the volatiles were removed in vacuum and the solid residue was redissolved in a THF:Py:Hexane mixture (1:1:2). Slow concentration of the solution at room temperature resulted in the formation of $\mathbf{1}(0.209 \mathrm{~g})$ in $86 \%$ yield.

Synthesis of $\left[\operatorname{Dy}\left(\mathrm{L}_{1}\right)_{2} \mathrm{Cl}(\mathrm{DME})\right] \cdot 0.5 \mathrm{DME}$ (2). A mixture of $\mathrm{HL}_{1}(0.200 \mathrm{~g}$, $0.566 \mathrm{mmol})$ and $\mathrm{KH}(0.029 \mathrm{~g}, 0.725 \mathrm{mmol})$ in THF $(30 \mathrm{~mL})$ was stirred at room temperature for $1 \mathrm{~h}$ and the resulting suspension was filtered. The clear yellow solution was added to a suspension of $\operatorname{DyCl}_{3}(0.076 \mathrm{~g}, 0.283$ $\mathrm{mmol})$ in THF $(10 \mathrm{~mL})$ and the reaction mixture was stirred overnight at room temperature. The solution was filtered, the volatiles were removed in vacuum and the solid residue was dissolved in DME. Slow concentration of the solution at room temperature resulted in the formation of 2 as yellow crystals. The mother liquid was decanted and the crystals were washed with cold hexane and dried in vacuum for 30 min. Complex 2 was isolated in $84 \%$ yield $(0.247 \mathrm{~g})$. Elemental analysis calcd. (\%) for $\mathrm{C}_{52} \mathrm{H}_{75} \mathrm{CIDyN}_{2} \mathrm{O}_{7}$ : C, 60.16; $\mathrm{H}, 7.28 ; \mathrm{N}, 2.70$; Dy, 15.65 ; found (\%): C, 60.50; H, 7.44; N, 2.36; Dy, 15.38. (Nujol, KBr) v/cm ${ }^{-1}: 500$ $(\mathrm{s}), 530(\mathrm{~m}), 610(\mathrm{~s}), 660(\mathrm{~s}), 710(\mathrm{~s}), 770(\mathrm{~s}), 840(\mathrm{~s}), 880(\mathrm{~m}), 920(\mathrm{~m})$, 980 (m), 1000 (s), 1050 (s), 1070 (s), 1155 (s), 1210 (s), 1260 (s), 1310 (s), $1415(\mathrm{~s}), 1540(\mathrm{~s}), 1620(\mathrm{~s})$.

Synthesis of $\left[\mathrm{Dy}\left(\mathrm{L}_{2}\right)_{2} \mathrm{Cl}\right] \cdot \mathbf{2 . 5}\left(\mathrm{C}_{7} \mathrm{H}_{8}\right)(3)$. A mixture of $\mathrm{HL}_{2}(0.200 \mathrm{~g}, 0.498$ $\mathrm{mmol})$ and $\mathrm{KH}(0.026 \mathrm{~g}, 0.646 \mathrm{mmol})$ in THF $(30 \mathrm{~mL})$ was stirred at room temperature for $1 \mathrm{~h}$ and the resulting suspension was filtered. The clear yellow solution was added to a suspension of $\mathrm{DyCl}_{3}(0.067 \mathrm{~g}, 0.249$ $\mathrm{mmol})$ in THF (10 mL) and the reaction mixture was stirred overnight at room temperature. The solution was filtered, the volatiles were removed in vacuum and the solid residue was dissolved in toluene. Slow concentration of the solution at room temperature resulted in the formation of 3 as yellow crystals. The mother liquid was decanted and the crystals were washed with cold hexane and dried in vacuum for 30 min. Complex 3 was isolated in $82 \%$ yield $(0.251 \mathrm{~g})$. Elemental analysis calcd. (\%) for $\mathrm{C}_{71.50} \mathrm{H}_{80} \mathrm{CIDyN}_{2} \mathrm{O}_{4}$ : C,69.85; H, 6.65; N, 2.27; Dy, 13.20; found (\%): C,69.47; H, 6.32; N, 2.01; Dy, 13.48. IR (Nujol, KBr) v/cm ${ }^{-1}$ : $480(\mathrm{~s}), 520(\mathrm{~m}), 610(\mathrm{~s}), 660(\mathrm{~s}), 730(\mathrm{~s}), 770(\mathrm{~s}), 840(\mathrm{~s}), 880(\mathrm{~m}), 920$ $(\mathrm{m}), 980(\mathrm{~m}), 1010$ (s), 1050 (s), 1070 (s), 1155 (s), 1210 (s), 1260 (s), $1310(\mathrm{~s}), 1415(\mathrm{~s}), 1540(\mathrm{~s}), 1580(\mathrm{~s}), 1625(\mathrm{~s})$.

\section{X-Ray crystallography}

X-ray diffraction data for 1-3 were collected on Bruker Apex II $(\mathbf{1}, \mathbf{3})$ and Bruker D8 Quest (2) diffractometers (graphite monochromator, $\omega$-scan technique) using Moka-radiation $(0.71073 \AA)$. The intensity data were integrated by SAINT program ${ }^{[32]}$ and were corrected for absorption and decay using SADABS. ${ }^{[33]}$ All structures were solved by direct methods and were refined by full-matrix least squares on F2 for all data using SHELX. ${ }^{[34]}$ All non-hydrogen atoms in 1-3 were found from Fourier syntheses of electron density and were refined anisotropically. All hydrogen atoms were placed in calculated positions and were refined as riding atoms with relative isotropic displacement parameters $\mathrm{U}(\mathrm{H})$ iso $=1.2 \mathrm{Ueq}\left(U(H)_{\text {iso }}=1.5 \mathrm{U}_{\text {eq }}\right.$ for methyl groups $)$. The structure of complex 2 was refined as a 2-component twin with TWIN $00-10-10-1$ 00 and $B A S F 0.38249$.

CCDC-1973040 (1), 1973041 (2) and 1973042 (3) contains the supplementary crystallographic data for this paper. These data are provided free of charge by The Cambridge Crystallographic Data Centre: ccdc.cam.ac.uk/structures. The crystallographic data and structure refinement details for 1-3 are given in Table S1.

\section{Magnetic Measurements}

Magnetic susceptibility data were collected with a Quantum Design MPMS-XL SQUID magnetometer working in the range $1.8-350 \mathrm{~K}$ with the magnetic field up to 7 Tesla. The samples were prepared in a glove box. The data were corrected for the sample holder and the diamagnetic contributions calculated from the Pascal's constants. The AC magnetic susceptibility measurements were carried out in the presence of a 3 Oe oscillating field in zero or applied external DC field.

\section{Photoluminescence measurements}

The emission and excitation spectra were recorded at $77 \mathrm{~K}$ and $295 \mathrm{~K}$ using a spectrofluorimeter Edinburgh FLS-920. The excitation source was a $450 \mathrm{~W}$ Xe arc lamp. The emission spectra were corrected for detection and optical spectral response of the spectrofluorimeter. Low temperature measurements $(77 \mathrm{~K})$ were performed using a liquid nitrogen dewar (quartz). The solid-state photoluminescence for $\mathrm{HL}_{1}$ and $\mathrm{HL}_{2}$ have been performed using an Ocean Optics USB2000 with an LED $(365 \mathrm{~nm})$ as the excitation source.

\section{Acknowledgements}

The authors thank the University of Montpellier, CNRS for financial support, Plateforme of Analysis and Characterisations (PAC) of ICGM for magnetic measurements. The Russian coauthors thank the Russian Science Foundation (grant 17-7330036).

\section{Conflict of Interest}

The authors declare no conflicts of interest.

Keywords: Lanthanides - Schiff bases $\cdot$ Magnetism $\cdot$ Single Molecule Magnets $\cdot$ Luminescence.

[1] a) D. N. Woodruff, R. E. P. Winpenny, R. A. Layfield, Chem. Rev. 2013 113, 5110-5148; b) S. T. Liddle, J. van Slageren, Chem. Soc. Rev. 2015, 44, 6655-6669; c) Z.-P. Ni, J.-L. Liu, M. N. Hoque, W. Liu, J.-Y. Li, Y.-C. Chen, M.-L. Tong, Coord. Chem. Rev. 2017, 335, 28-43; d) Z. Zhu, M. Guo, X.-L. Li, J. Tang, Coord Chem. Rev. 2019, 378, 350-364.

[2] a) J. Luzon, R. Sessoli, Dalton Trans. 2012, 41, 13556-13567; b) F. Troiani, M. Affronte, Chem. Soc. Rev. 2011, 40, 3119-3129; c) L. Bogani, W. Wernsdorfer, Nat. Mater. 2008, 7, 179-186.

[3] a) J. D. Rinehart, J. R. Long, Chem. Sci. 2011, 2, 2078-2085; b) L. Ungur, L. F. Chibotaru, Inorg. Chem. 2016, 55, 10043-10056.

[4] a) C. A. P. Goodwin, F. Ortu, D. Reta, N. F. Chilton, D. P. Mills, Nature 2017, 548, 439-442; b) L. Escalera-Moreno, J. J. Baldoví, A. GaitaAriño, E. Coronado, Chem. Sci. 2018, 9, 3265-3275; c) A. Lunghi, F. Totti, R Sessoli, S Sanvito, Nat Comm 2017, 8, 14620; d) M. J. Giansiracusa, A. K. Kostopoulos, D. Collison, R. E. P. Winpenny, N. F Chilton, Chem. Commun. 2019, 55, 7025-7028; e) P. Evans, D. Reta G. F. S. Whitehead, N. F. Chilton, D. P. Mills, J. Am. Chem. Soc. 2019 $141,19935-19940$.

[5] a) F.-S. Guo, B. M. Day, Y.-C. Chen, M.-L. Tong, A. Mansikkamäki, R. A. Layfield, Angew. Chem. Int. Edit. 2017, 56, 11445-11449; b) F.-S. Guo, B. M. Day, Y.-C. Chen, M.-L. Tong, A. Mansikkamäki, R. A Layfield, Science 2018, 362, 1400-1403.

[6] a) J. Liu, Y.-C. Chen, J.-L. Liu, V. Vieru, L. Ungur, J.-H. Jia, L. F. Chibotaru, Y. Lan, W. Wernsdorfer, S. Gao, X.-M. Chen, M.-L. Tong, J. Am. Chem. Soc. 2016, 138, 5441-5450; b) S. K. Gupta, T. Rajeshkumar, G. Rajaraman, R. Murugavel, Chem. Sci. 2016, 7, 51815191 ; c) F. S. Guo, B. M. Day, Y. C. Chen, M. L. Tong, A. Mansikkamaki, R. A. Layfield, Angew. Chem. Int. Ed. Engl. 2017, 56 11445-11449; d) Y.-S. Ding, N. F. Chilton, R. E. P. Winpenny, Y.-Z. Zheng, Angew. Chem. Int. Edit. 2016, 55, 16071-16074; e) Y.-S. Meng 
L. Xu, J. Xiong, Q. Yuan, T. Liu, B.-W. Wang, S. Gao, Angew. Chem. Int. Edit. 2018, 57, 4673-4676.

[7] N. F. Chilton, Inorg. Chem. 2015, 54, 2097-2099.

[8] T. Pugh, N. F. Chilton, R. A. Layfield, Angew. Chem. Int. Edit. 2016, 55, 11082-11085.

[9] a) Y.-C. Chen, J.-L. Liu, L. Ungur, J. Liu, Q.-W. Li, L.-F. Wang, Z.-P. Ni, L. F. Chibotaru, X.-M. Chen, M.-L. Tong, J. Am. Chem. Soc. 2016, 138 2829-2837; b) Y. S. Ding, T. Han, Y. Q. Zhai, D. Reta, N. F. Chilton, R E. P. Winpenny, Y. Z. Zheng, Chem. Eur. J. 2020, 26, 5893-5902.

[10] a) J. Tang, P. Zhang, in Lanthanide Single Molecule Magnets, 10.1007/978-3-662-46999-6 2, Springer Berlin Heidelberg, Berlin, Heidelberg, 2015, pp. 41-90; b) R. A. Layfield, M. Murugesu, Lanthanides and Actinides in Molecular Magnetism, Wiley, 2015; c) J. Long, M. S. Ivanov, V. A. Khomchenko, E. Mamontova, J.-M. Thibaud J. Rouquette, M. Beaudhuin, D. Granier, R. A. S. Ferreira, L. D. Carlos, B. Donnadieu, M. S. C. Henriques, J. A. Paixão, Y. Guari, J. Larionova, Science 2020, 367, 671.

[11] a) M. Andruh, Dalton Trans. 2015, 44, 16633-16653; b) J. Long, Y. Guari, R. A. S. Ferreira, L. D. Carlos, J. Larionova, Coord. Chem. Rev. 2018, 363, 57-70; c) J. Long, Frontiers in Chemistry 2019, 7, 63; d) J.H. Jia, Q.-W. Li, Y.-C. Chen, J.-L. Liu, M.-L. Tong, Coord. Chem. Rev. 2019, 378, 365-381.

[12] a) I. Oyarzabal, J. Ruiz, E. Ruiz, D. Aravena, J. M. Seco, E. Colacio Chem. Commun. 2015, 51, 12353-12356; b) J. P. Costes, S. TitosPadilla, I. Oyarzabal, T. Gupta, C. Duhayon, G. Rajaraman, E. Colacio, Chem. Eur. J. 2015, 21, 15785-15796; c) Z.-H. Li, Y.-Q. Zhai, W.-P Chen, Y.-S. Ding, Y.-Z. Zheng, Chem. Eur. J. 2019, 25, 16219-16224; d) A. B. Canaj, S. Dey, E. R. Martí, C. Wilson, G. Rajaraman, M. Murrie, Angew. Chem. Int. Edit. 2019, 58, 14146-14151.

[13] J. Long, I. V. Basalov, N. V. Forosenko, K. A. Lyssenko, E. Mamontova A. V. Cherkasov, M. Damjanović, L. F. Chibotaru, Y. Guari, J. Larionova, A. A. Trifonov, Chem. Eur. J. 2019, 25, 474-478.

[14] D. Casanova, M. Llunell, P. Alemany, S. Alvarez, Chem. Eur. J. 2005 $11,1479-1494$.

[15] N. F. Chilton, D. Collison, E. J. L. Mclnnes, R. E. P. Winpenny, A. Soncini, Nat. Commun. 2013, 4, 2551.

[16] J. Long, A. N. Selikhov, E. Mamontova, K. A. Lyssenko, Y. Guari, J. Larionova, A. Trifonov, Dalton Trans. 2020, 49, 4039-4043.

[17] K. R. Meihaus, S. G. Minasian, W. W. Lukens, S. A. Kozimor, D. K Shuh, T. Tyliszczak, J. R. Long, J. Am. Chem. Soc. 2014, 136, 6056 6068.

[18] K. N. Shrivastava, physica status solidi (b) 1983, 117, 437-458

[19] a) P. L. Scott, C. D. Jeffries, Phys. Rev. 1962, 127, 32-51; b) A Abragam, B. Bleaney, Electron paramagnetic resonance of transition ions, Oxford : Clarendon press, 1970

[20] J. H. Van Vleck, Phys. Rev. 1940, 57, 426-447.

[21] A. Chiesa, F. Cugini, R. Hussain, E. Macaluso, G. Allodi, E. Garlatti, M. Giansiracusa, C. A. P. Goodwin, F. Ortu, D. Reta, J. M. Skelton, T. Guidi, P. Santini, M. Solzi, R. De Renzi, D. P. Mills, N. F. Chilton, S. Carretta, Phys. Rev. B 2020, 101, 174402.

[22] a) L. T. A. Ho, L. F. Chibotaru, Phys. Rev. B 2018, 97, 024427; b) Y.-S Ding, K.-X. Yu, D. Reta, F. Ortu, R. E. P. Winpenny, Y.-Z. Zheng, N. F. Chilton, Nat. Comm. 2018, 9, 3134.

[23] D. Reta, N. F. Chilton, Phys. Chem. Chem. Phys. 2019, 21, 23567 23575.

[24] D. Gatteschi, R. Sessoli, J. Villain, Molecular nanomagnets, Vol. 5, Oxford University Press on Demand, 2006.

[25] M. Gregson, N. F. Chilton, A.-M. Ariciu, F. Tuna, I. F. Crowe, W. Lewis A. J. Blake, D. Collison, E. J. L. Mclnnes, R. E. P. Winpenny, S. T. Liddle, Chem. Sci. 2016, 7, 155-165.

[26] J.-L. Liu, Y.-C. Chen, M.-L. Tong, Chem. Soc. Rev. 2018, 47, 2431 2453.

[27] a) F. Habib, G. Brunet, V. Vieru, I. Korobkov, L. F. Chibotaru, M. Murugesu, J. Am. Chem. Soc. 2013, 135, 13242-13245; b) K. Liu, H. Li, X. Zhang, W. Shi, P. Cheng, Inorg. Chem. 2015, 54, 10224-10231; c) W. Chin, P.-H. Lin, Inorg. Chem. 2018, 57, 12448-12451; d) J. Long, D. Lyubov, T. Mahrova, A. Cherkasov, G. K. Fukin, Y. Guari, J. Larionova A. Trifonov, Dalton Trans. 2018, 47, 5153-5156; e) S. Yu, Z. Chen, H. Hu, B. Li, Y. Liang, D. Liu, H. Zou, D. Yao, F. Liang, Dalton Trans. 2019, 48, 16679-16686; f) S. P. Petrosyants, K. A. Babeshkin, A. V. Gavrikov, A. B. Ilyukhin, E. V. Belova, N. N. Efimov, Dalton Trans. 2019, 48 12644-12655; g) Z. Chen, S. Yu, R. Wang, B. Li, B. Yin, D. Liu, Y Liang, D. Yao, F. Liang, Dalton Trans. 2019, 48, 6627-6637; h) K.-X. Yu, Y.-S. Ding, Y.-Q. Zhai, T. Han, Y.-Z. Zheng, Dalton Trans. 2020, 49, 3222-3227.

[28] a) D. H. Moseley, S. E. Stavretis, K. Thirunavukkuarasu, M. Ozerov, Y. Cheng, L. L. Daemen, J. Ludwig, Z. Lu, D. Smirnov, C. M. Brown, A Pandey, A. J. Ramirez-Cuesta, A. C. Lamb, M. Atanasov, E. Bill, F. Neese, Z.-L. Xue, Nat. Comm. 2018, 9, 2572-2572; b) D. H. Moseley, S. E. Stavretis, Z. Zhu, M. Guo, C. M. Brown, M. Ozerov, Y. Cheng, L. L. Daemen, R. Richardson, G. Knight, K. Thirunavukkuarasu, A. J. Ramirez-Cuesta, J. Tang, Z.-L. Xue, Inorg. Chem. 2020, 59, 5218-5230.

[29] K. Randall McClain, C. A. Gould, K. Chakarawet, S. J. Teat, T. J. Groshens, J. R. Long, B. G. Harvey, Chem. Sci. 2018, 9, 8492-8503.
[30] P. A. Cameron, V. C. Gibson, C. Redshaw, J. A. Segal, A. J. P. White, D. J. Williams, J. Chem. Soc., Dalton Trans. 2002, 10.1039/B106131N, 415-422.

[31] S. J. Lyle, M. M. Rahman, Talanta 1963, 10, 1177-1182.

[32] Bruker, (Ed.: B. A. Inc.), Madison, Winsconsin, USA, 2018.

[33] L. Krause, R. Herbst-Irmer, G. M. Sheldrick, D. Stalke, J. Appl. Crystallogr. 2015, 48, 3-10.

[34] G. Sheldrick, Acta Cryst. C. 2015, 71, 3-8. 
